\title{
LA REPRÉSENTATION DU COUPLE DANS QUELQUES ROMANS QUÉBÉCOIS RÉCENTS
}

Irène Oore, Dalhousie University

Au cœur même de toute problématique relationnelle se trouve le couple. Car le couple est l'unité minimale de la société, unité qui telle une molécule présente les caractéristiques de l'organisme (ici de la société) au sein de laquelle elle évolue. D'autre part il s'agit d'une unité qui subit les influences de la société qui I'entoure et semble se transformer en fonction de cette société.

Nous avons entrepris d'examiner le couple dans une dizaine de romans écrits durant une décennie (entre 1993 et 2003). Nous voulions étudier la représentation du couple dans le roman québécois récent. Toutefois, nous voulions que notre corpus puisse être examiné avec une perspective et un recul critiques. Aussi le roman le plus récent que nous examinons date-t-il de 2002.

Afin d'assurer un corpus représentatif nous avons choisi délibérément des romans écrits tant par des femmes (6) que par des hommes (4), tant par des écrivains migrants (4) que par les écrivains « de souche »(6), romans écrits de plusieurs points de vue aussi bien intradiégétiques qu'extradiégétiques ${ }^{1}$ : celui d'un narrateur masculin à la $1^{\text {re }}$ personne $(2)$, celui d'un point de vue d'un narrateur féminin à la $1^{\text {re }}$ personne (2), celui d'un point de vue de narrateurs multiples à la $1^{\text {re }}$ personne (3) et d'un point de vue d'un narrateur (plus ou moins omniscient) à la $3^{\mathrm{e}}$ personne (3).

Nous avons retenu dans I'ordre chronologique de leur parution Les lettres chinoises de Ying Chen $(1993 \text {, réédité en } 1999)^{2}$, Unless d'Hélène Monette $(1995)^{3}$, Lîle de la Merci d’Élise Turcotte $(1997)^{4}$, La vengeance d'un père de Pan Bouyoucas $(1997)^{5}$, Le bonheur a la queue glissante d'Abla Farhoud $(1998)^{6}$, Le bout de la terre de Yan Muckle $(1998)^{7}$, Un sourire blindé de Sergio Kokis $(1998)^{8}$, La danse juive de Lise Tremblay $(1999)^{9}$, Les vents dominants de Jacques Marchand (1999) ${ }^{10}$ et Soudain le Minotaure de Marie Hélène Poitras $(2002)^{11}$.

\section{Les trois « âges » du couple}

Dans chacun des textes étudiés, nous retrouvons, intimement liés à la notion du couple, l'amour (ou du moins le désir de l'amour) et la violence (qu'elle soit verbale ou physique).

Dans ces romans plusieurs couples se forment, se défont, se reconstituent (avec les mêmes ou d'autres partenaires). Clairement, le couple est représenté comme une unité dynamique et nous ne parviendrons qu'à examiner certaines de ses caractéristiques, quelques-uns de ses défis et des réponses que les romans proposent à ces défis.

http://www.brocku.ca/cfra/voixplurielles06-01/index.html 
D’ailleurs il faut noter que les caractéristiques, et surtout les préoccupations du couple, varient avec l'âge des partenaires.

\section{Les adolescents}

Dans L'île de la Merci d'Élise Turcotte, nous observons un couple d'adolescents, Hélène et Thomas, dont la préoccupation majeure est la sexualité. Ils constituent un couple hésitant et maladroit qui vit une première expérience sexuelle gauche et peu satisfaisante. Une fois l'acte accompli, la raison d'être de ce couple disparaît et le couple se défait. Dans Le bout de la terre de Yan Muckle nous observons le narrateur adolescent, Alex, avec Michèle, une jeune femme de quatre ans son aînée, vivre ensemble pendant un an. Le couple se sépare : la rupture semble due en grande partie à l'incertitude qu'éprouve Alex quant à la direction à prendre dans sa vie, incertitude propre à l'adolescence. Deux autres couples, Dominique Soucy et Gabriel Baltas (deux adolescents) dans La vengeance d'un père de Bouyoucas, Red (une adolescente) et Fernand (un adulte) dans Unless de Monette, se comportent en personnes authentiques et responsables. Ici ce sont, tristement, les interférences de la société qui rendent problématique la survie du jeune couple. Dans Unless, la travailleuse sociale, le juge et la bureaucratie se mêlent de cela; dans La vengeance d'un père, c'est un criminel psychopathe qui rompt le cours de la vie de Dominique et ses chances de mener une vie normale.

\section{Les adultes}

Les couples d'adultes dans les romans à l'étude abondent. Souvent leurs préoccupations tournent autour des moyens de gagner leur vie, de leur travail et de leur famille. Plusieurs parmi eux sont divorcés et quelques-uns ont un nouveau partenaire. Unless perd son travail, Yannis (VP) doit s'occuper de sa boulangerie alors qu'il traverse avec sa famille une crise bien grave, Scott dans Les vents dominants part de Seattle à Montréal afin de préparer le tournage d'une série télévisée. Enfin Robert et Viviane dans Lîle de la Merci sont bien inquiets, Viviane parce que les maisons ne se vendent pas et Robert parce que les ordinateurs ne se vendent pas non plus (IM 142). Cette inquiétude ajoute à la tension insupportable au sein du couple.

Il est particulièrement intéressant de noter à propos de ces couples d'adultes que les romans à l'étude, reflétant en cela sans doute la réalité, présentent les parents des narrateurs ou des jeunes protagonistes (adolescents ou adultes) comme des couples divorcés. C'est le cas des parents de la narratrice dans $L a$ danse juive, des parents du narrateur Guillaume dans Les vents dominants, de ceux d'Alex, dans Le bout de la terre ainsi que de Dominique Soucy dans La vengeance d'un père ${ }^{12}$. Lorsqu'ils ne sont pas divorcés, I'un des parents a disparu; c'est le cas d'Adélaïde, la femme de Walter dans Unless; c'est aussi le cas de Dario, le mari de Natividad dans Un sourire blindé. Robert et Viviane dans Lîle de la Merci semblent tellement tendus et malheureux que leurs filles, Hélène et Viviane, angoissées, se demandent si l'un d'eux est sur le

http://www.brocku.ca/cfra/voixplurielles06-01/index.html 
point de partir: «Et les voilà qui se mettent à énumérer une série de choses plus graves et plus importantes que l'éventuel départ d'un de leurs parents » (IM 173).

\section{Les vieux couples}

Dans Unless et dans Le bonheur a la queue glissante nous observons deux vieux couples. Walter et Léonie ne sont pas mariés l'un à l'autre. Chacun d'eux a été marié de son côté. Ils sont décrits par les narratrices, filles de Walter. Milou est passablement charitable : « J'ai même vu Léonie. Pas mal jolie, la madame! Walter est aux petits soins avec elle. C'est beau de les voir » (U 18). Ce qui n'est pas le cas d'Unless. Selon Unless, Léonie est une intruse qui ne fait pas partie de la famille : « Mais qu'est-ce qu'elle fait ici, celle-là? » (U 59); et ailleurs Unless ricane : «Des petites bouchées volées au temps saccagé de sa vieillesse. Avec Léonie qui cuisine. Il s'est remis à jouer au bowling. Avec Léonie sur le banc. Il est allé souper chez Milou. Avec Léonie qui ne boit pas » (U 85).

Salim et Dounia, nés au Liban, se sont mariés jeunes, ont élevé leurs enfants et ont passé ensemble leur vieillesse jusqu'à la mort de Salim. Ce couple est décrit du point de vue de Dounia. De par leur background culturel, de par le fait que Dounia ne parle pas français, ne sait ni lire ni écrire, le divorce n’a pas été une véritable option. Pourtant, elle avoue y avoir pensé. Elle n’a jamais été heureuse en couple. Elle s'y est résignée. Vers la toute fin de sa vie, dans une espèce de rêve-délire, Dounia s'imagine Salim I'invitant tendrement à prendre un café en tête-à-tête (BQG 165). Ce geste pourtant si simple, si facile, si fréquent chez d'autres, ne s'est jamais produit chez ce couple.

Il ne s'agit que d'un survol rapide des couples des romans à divers moments de leur vie; il semble que les préoccupations des couples varient en effet selon leur âge, toutefois nous croyons que certains traits, certaines façons d'être, peuvent contribuer au malaise ou au bien-être du couple à chacune des étapes de sa vie. Nous tâcherons de dégager ces forces telles qu'elles se présentent dans les romans à l'étude.

\section{Les forces négatives}

\section{Précarité}

La caractéristique prédominante du couple dans le roman québécois récent semble être sa précarité : l'avenir de ce couple n'est guère assuré. On semble trouver dans les romans plus de couples divorcés que de couples mariés; pourtant la grande majorité des couples représentés n'est ni mariée ni divorcée. Seuls deux couples sont fiancés dans les dix romans. Il est pertinent peut-être de noter qu'il s'agit de deux couples dont la culture d'origine n'est pas québécoise, car les fiançailles, ainsi que le mariage d'ailleurs, semblent être en voie de disparition au Québec. Les fiançailles des deux couples en question sont rompues dans chacun des cas.

http://www.brocku.ca/cfra/voixplurielles06-01/index.html 
Yuan et Sassa (LC) se sont fiancés en Chine avant le départ de Yuan pour Montréal. Cette promesse solennelle de mariage leur paraît importante, du moins au début; d'ailleurs il semble que cette promesse ait plus d'importance en Chine qu'au Québec. Da Li, une amie chinoise de Yuan et de Sassa travaille à Montréal dans un restaurant et elle note dans une lettre adressée à Sassa : « Ce qui me frappait le plus, c'était que nos clients changeaient sans cesse d'amie de fille ou d'ami de garçon ou d'épouse ou d'époux » (LC 40). À la fin du roman Sassa qui croit que son fiancé la trompe, rompt avec lui. La promesse des fiançailles n'est pas assez solide pour garder intact ce couple. D'ailleurs, le phénomène d'inconstance semble être présent en Chine aussi : Sassa accuse sa sœur de sortir avec deux garçons à la fois (LC 59). Enfin, Sassa caractérise les amours de Da Li comme des amours sans suite : «Beaucoup flottaient en l'air comme des chansons. Elles s'élevaient et descendaient, changeantes, sans but ni promesse» (LC 53). Cette description correspond parfaitement au caractère précaire des rapports de la plupart des couples dans les romans examinés.

Le second couple fiancé est celui de Tony Baltas et d'Éléna Fokas dans La vengeance d'un père (les parents de I'un et de l'autre ont immigré au Québec de la Grèce). Il s'agit d'un couple peu stable; Éléna n'aime pas que son fiancé s'occupe du trafic de drogue la nuit du jour de l'An et elle le provoque en dansant et en s'embrassant avec un autre devant tous leurs amis. Le couple ne se réconciliera jamais. À la fin du roman Éléna se suicide.

La précarité du couple est évidente dans Un sourire blindé; Natividad, la mère de Conrado est seule; Dario, le père de l'enfant l'a quittée. Elle le remplace par d'autres «papas »: il s'agit d'un véritable défilé d'hommes qui arrivent, font la fête et disparaissent: «Camilo, Zenobio, Guillerme, Pierre, Luciano... Conrado perdait le compte et commençait à les confondre » (SB 67). Conrado apprend que «plusieurs d'entre eux étaient mariés ou avaient des enfants quelque part [...] (SB 68). La précarité, l'impermanence, I'instabilité et la légèreté marquent tous ces couples. C'est Conrado et les autres enfants nés de ces couples qui souffrent le plus de cette absence de repères, de cette instabilité perturbante.

\section{Toxicomanie, alcoolisme et violence}

Dans cinq des dix romans l'alcool et les drogues jouent un rôle; il s'agit bien évidemment d'une façon de s'évader d'une réalité difficile et d'une tentative d'apaiser la souffrance. Toutefois l'alcool et la drogue semblent contribuer directement ou indirectement à la séparation du couple et à la désagrégation de la famille.

Dans Unless Chut et Red boivent et se droguent. Chut, dont la petite amie Manon le quitte, se suicide, alors que Red se prostitue afin de s'acheter de la drogue. C'est seulement lorsqu'elle arrête de se droguer que Red aura une relation qui promet d'être stable. Dans La vengeance d'un père le détective Georges Soucy est séparé de sa femme Louise parce que cette dernière est alcoolique. Son ressentiment et sa 
rancœur envers Louise sont grands. Toujours dans ce roman, comme nous l'avons déjà noté, Tony Baltas est impliqué dans le trafic de drogue et une autre jeune, Anaïs Gauthier, est héroïnomane; comme Red dans Unless, Anaïs elle aussi se prostitue afin de pouvoir se payer les drogues dont elle a besoin. Afin de les obtenir Anaïs est prête à trahir son partenaire et révèle les secrets de celui-ci au détective. L'alcool et les drogues font partie intégrante de la vie instable de Natividad et de ses nombreux partenaires dans Un sourire blindé. Enfin, Mel, le partenaire de la narratrice de La danse juive, boit. C'est cette dépendance qui rendra son échec irréversible.

Associée parfois à l'alcool et à la drogue, la violence est présente dans chacun des romans examinés, mais c'est le nombre de viols, de meurtres et de suicides dans ces romans que nous avons trouvé surprenant; ce nombre dépasse de loin le nombre auquel on aurait pu s'attendre en choisissant au hasard une dizaine de romans québécois d'une autre décennie. Les romanciers font-ils preuve d'une simple soif du sensationnalisme des médias? Ou encore croient-ils répondre au goût de leurs lecteurs de plus en plus désensibilisés? Quel que soit le cas, nous croyons que ce phénomène reflète la peur profonde dont la société est imprégnée.

Bien que certains actes de violence ne semblent être directement associés au couple, ils contribuent à la création d'une ambiance violente qui ne peut qu'influencer le couple. Souvent les victimes de la violence sont encore les femmes, même dans le roman québécois des années 1990. Dans Unless Chut se suicide et Mona, une jeune fille de dix-sept ans, est assassinée et abandonnée dans le fossé (U 148). Une autre jeune fille, Marie-Pierre Sauvé, est violée et tuée dans Lîile de la Merci; cet acte de violence extrême hante et pèse sur la vie de tous les protagonistes. Dounia dans Le bonheur a la queue glissante a subi la violence tant verbale que physique de son mari Salim; vers la fin de sa vie elle se reproche de ne pas I'avoir tué! (BQG 152). Alex, le narrateur du Bout de la terre est témoin d'une femme brutalisée, battue et violée par un homme. Alex intervient, est agressé lui aussi, et avant de partir la femme explique à Alex que I'homme est « le mari de sa sœur » (BT 152-153). Dans Un sourire blindé le petit Conrado voit son père battre sa mère, puis faire exploser la télévision. Conrado lui-même, quelques années plus tard, finit par tuer un homme qui abuse d'une enfant. Dans Soudain le Minotaure, Mino Torrès viole les femmes. Pour lui le viol est une expression de rage, il s'agit d'« enfoncer [s]a rage dans des corps de filles » (SLM 60). C'est peut-être Guillaume, le narrateur des Vents dominants, qui explique le mieux ce que la violence offre à l'agresseur; ayant tué Paule il remarque que ce meurtre les unit d'une façon irréversible. Le meurtre offre la permanence à une liaison qui autrement aurait été insignifiante et brève pour Paule; Guillaume conclut : «Tu ne pouvais plus te réfugier ailleurs qu'en moi : nous étions liés désormais de manière indissoluble » (VD 139). La violence qui semble découler de la précarité, constitue une réponse à celle-ci.

\section{Le triangle}

http://www.brocku.ca/cfra/voixplurielles06-01/index.html 
Si le couple peut espérer atteindre des rapports harmonieux à deux, la structure triangulaire, vieille comme le monde et très présente dans le roman québécois récent, n'annonce rien de bon. Le triangle, réel ou imaginaire, composé de deux hommes et d'une femme ou de deux femmes et d'un homme ${ }^{13}$, est une source de tensions et d'une souffrance souvent insupportable au sein du couple ${ }^{14}$. Le triangle précipite la désintégration du couple. La trahison, la culpabilité, la jalousie et la perte de confiance détruisent le couple. Dans Les lettres chinoises le fait que Yuan semble tromper sa fiancée Sassa avec leur amie Da Li provoque la rupture des fiançailles. Quant à Unless et Sherpa, afin d'arrêter de souffrir et de faire souffrir Sherpa, il faut qu'Unless arrête d'imaginer Magali avec Sherpa : «J'essayais de ne plus imaginer Magali en Vénus de Sherpa » (U 138). Dans Le bonheur a la queue glissante Dounia décrit aussi sa jalousie irraisonnée de son mari Salim : «Juste à la pensée que Salim pouvait parler à une autre femme, la toucher, rire avec elle, j'étais torturée » (BQG 62). Alex, le narrateur du Bout de la terre aime Sarah, or Sarah a été dans le passé avec leur ami Pietro; Alex est jaloux de ce passé immuable que Pietro et Sarah partagent, il en souffre et se sent impuissant à le dépasser : «Je ne pouvais pas faire comme si cela n'avait jamais eu lieu » (BT 64). Notons que parfois le «triangle » n'est qu'une manifestation d'un malaise plus profond au sein du couple. C'est le cas d'Éléna et de Tony dans La vengeance d'un père. Afin de « se venger » Éléna flirte avec Julien...

Scott et Françoise, les parents de Guillaume (le narrateur dans Les vents dominants), ont divorcé lorsque Guillaume avait six ans. Guillaume soupçonne que c'était à cause d'une liaison de Scott avec Paule. À seize ans, alors qu'il rend visite à Paule avec Scott et sa femme Brenda, Guillaume devient I'amant de Paule (se crée ainsi le triangle classique évoquant le mythe de Phèdre et le fameux triangle de Phèdre, de Thésée et d'Hippolyte). Guillaume tue son amante Paule, vengeant ainsi sans jamais (se) le dire, sa mère. Les vents dominants sont le drame moderne fondé essentiellement sur des structures triangulaires et les jeux dramatiques et tragiques qui les accompagnent. Scott-Brenda-Françoise, Scott-Brenda-Guillaume, Scott-Guillaume-Paule, Françoise-Guillaume-Paule, véritable kaléidoscope aux images triangulaires. Enfin, dans La danse juive, la narratrice évoque la structure triangulaire comme un cliché mélodramatique qui réussit à vendre des programmes télévisés à un public cible avide du kitsch qu'on lui offre : « L'image s'impose. Une femme, ma mère, se lève à l'arrière de la salle, la caméra fait un gros plan. La femme est trop maquillée, elle porte un chandail tissé de fils d'argent qui font des reflets désagréables à l'écran. Elle dit que son mari l'a quittée pour une femme plus jeune, l'émotion monte, elle retient un peu ses larmes [...] » (D) 11-12).

\section{Le jeu du bourreau, de la victime et du sauveteur}

Afin de pouvoir atteindre une certaine sérénité, le couple doit éviter tout jeu inauthentique où les rôles sont prédéterminés, dramatisés, exagérés et fondamentalement faux. Il s'agit du jeu du bourreau, de la victime et du sauveteur, jeu à structure triangulaire ${ }^{15}$. Un personnage peut jouer à divers moments 
différents rôles, il peut être à la limite son propre bourreau et sa propre victime. L'intervention d'un sauveteur n'est pas toujours fausse. Il ne s'agit pas toujours d'un jeu. Parfois, au contraire, il s'agit d'un acte profondément humain. Lorsque Red traverse une crise où elle n'arrête pas de se détruire, de se tuer, Fernand intervient en véritable sauveteur. Red raconte : «Au lieu de l'envoyer promener, je lui dis que depuis que j'ai craqué, je ne sais que mourir. Il dit que c'est pas vrai, que je veux vivre » (U 171). C'est en grande partie grâce à Fernand que Red s'en sortira : «Fernand mon ami, il regarde ma guerre, les bateaux sur le fleuve, et il défie une à une les flammes de l'enfer qui me laissent torche vivante dans ses bras de pompier » (U 170-171). Le rôle du sauveteur est approprié pour le moment, mais Fernand I'abandonnera dès qu'il ne sera plus nécessaire. Ce qui nous intéresse ici, ce sont les rôles superflus joués au sein d'un couple, rôles qui faussent les rapports et rendent impossible toute communication véritable, car ces rôles confinent les acteurs à un script prévisible, leur ôtant liberté et autonomie. Dans Le bout de la terre, Alex, le narrateur, joue le rôle du bourreau envers Michèle. Michèle d'autre part, joue la victime. Bien qu'ils se complètent dans leurs rôles, ils sont tous les deux malheureux et s'acheminent inéluctablement vers la rupture. Voici comment Alex décrit leur scénario récurrent : « Je lui reprochais sa naïveté. Moi qui a priori ne connaissais rien au métier de comédien ("c'est mon métier, criait-elle pour me faire taire, mon métier!"), je I'accusais de se contenter de singer [...] Le ton montait [...] Cela finissait toujours de la même façon : elle s'effondrait, prête à se trancher la tête ou à se métamorphoser en tapis, plus convaincue que jamais d'être une incurable nullité, alors que je me maudissais d'être ce bourreau cruel [...] 》 (BT 21) ${ }^{16}$.

Nous trouvons un autre exemple de ce script incontournable, et dont la structure est parfaitement triangulaire dans La vengeance d'un père. Georges Soucy, le détective, a assisté, enfant, à des scènes où son père maltraitait sa mère. Le père jouait le rôle du bourreau et la mère celui de la victime. Le fils, fatigué de ce jeu, a intervenu (en tant que sauveteur) : «n'en pouvant plus de voir son père la maltraiter [Soucy] avait traité ce dernier de "boss des bécosses" » (VP 147) et il a été sidéré par la réaction de sa mère : «Plutôt que d'appuyer son fils, elle avait défendu le mari qui avait fait de sa vie un calvaire. Soucy en avait été tellement écœuré que, quelques années plus tard, quand, se mourant du cancer, elle lui avait demandé de la pardonner de lui avoir gâché, par sa soumission, une belle partie de son enfance, il avait refusé » (VP 147). Dans ce cas la victime (la mère de Soucy) ne veut pas être sauvée et le sauveteur en est profondément vexé. Lorsque sa mère lui demande pardon, c'est son fils, Soucy, qui à son tour, devient le bourreau...

Enfin le roman Soudain le Minotaure est entièrement basé sur le script prédateur-proie (ou bourreauvictime) et le mythe de Minotaure et d'Ariane. Mino Torres qui a violé des dizaines de femmes, écrit en prison: «Si l'homme a envie de violer, [la femme] lui donnera l'impression de n'être bonne qu'à ça. D'attendre ça même » (SLM 17). D'ailleurs selon ce prédateur, l'invitation au jeu bourreau-victime vient 
souvent de la proie. Voici comment il présente cela : «Cette fille était une invitation au viol, un présage des autres agressions à venir. En jouant la proie, elle avait réveillé le prédateur en moi » (SLM 34). Or, Ariane, sa dernière victime et narratrice, elle aussi, a refusé de jouer son rôle de victime et le jeu a été interrompu.

Il est bien intéressant de noter que Mino est très conscient qu'il participe à un jeu : « Le viol est un jeu. Il faut trouver les bons joueurs, sinon tout cloche. C'est un jeu de rôle » (SLM 81).

\section{Les forces positives au sein du couple}

Puisque le couple dans les romans québécois à l'étude semble précaire et malheureux, puisque la toxicomanie, I'alcoolisme, la dépression et la violence dominent, afin de cerner les forces positives au sein du couple nous avons décidé d'examiner quelques couples que les romans nous proposent comme modèles. A partir de cet examen nous espérons pouvoir mieux cerner les forces positives qui permettent aux couples privilégiés des moments de bonheur quelque fugitifs qu'ils soient. Les couples que nous examinons ici semblent s'aimer et leurs rapports paraissent sains. Ils refusent les jeux inauthentiques et cruels des couples malheureux.

\section{Le frère de la narratrice et sa femme (La danse juive)}

La narratrice de La danse juive n'est pas une femme heureuse. Son point de vue est particulièrement critique face au mythe du couple heureux véhiculé par les médias, et notamment par les magazines et la télévision. Elle prend un plaisir douloureux à dénoncer ce mensonge : « Sur l'une des premières pages [d'un magazine], mon père pose fièrement avec sa nouvelle flamme. Derrière eux, une maison blanche avec une véranda. Le kitsch américain dans toute sa splendeur » (DJ 13). Selon la narratrice il s'agit là d'une fausse façade. Alors que ce scepticisme semble justifié dans le cas du père, on se demande dans quelle mesure la narratrice est équitable envers son frère et sa belle-sœur. La narratrice admet ne pas être attachée à sa nièce (DJ 68) et elle se souvient de ses rapports tumultueux avec son frère lorsqu'ils étaient adolescents : « Les deux adolescents violents qui se rouaient de coups dans leur sous-sol luxueux [...] (D] 72). L'histoire du frère et de sa femme est simple : tous les deux ingénieurs, ils se sont rencontrés à l'université, ils ont emménagé dans un sous-sol où ils sont restés jusqu'à la fin de leurs études (DJ 68). Ils ont une enfant dont tous les deux s'occupent. Tous les deux ont accepté un contrat de deux ans dans le sud de la Saskatchewan, près de Regina, et ils montrent des photos des maisons de la banlieue qu'ils habiteront. La narratrice reproche à son frère sa vie rangée, son conformisme (DJ 95). Ce frère qui semble raisonnable et équilibré, qui mène une vie de couple parfaitement stable depuis longtemps, ne choque point le lecteur. En fait, l'irritation de la narratrice semble mettre en relief ses frustrations personnelles plutôt qu'autre chose. Aussi avons-nous inclus ce couple conventionnel, raisonnable et solide parmi les couples «modèles » malgré le point de vue de la narratrice, tout en admettant que nous avons peu de renseignements sur ce couple. Néanmoins, il nous semble qu'ils ont su dès leur première rencontre qu'ils voulaient être ensemble

http://www.brocku.ca/cfra/voixplurielles06-01/index.html 
et ils avancent ensemble dans la vie ayant des rêves (il est vrai que ces rêves sont conventionnels), faisant des plans et les exécutant ${ }^{17}$.

\section{Unless et Sherpa (Unless)}

Nous suivons Unless et Sherpa à travers les hauts et les bas d'un couple qui semble malgré tout bien assorti à certains égards : il y a entre eux beaucoup d'affection, une grande camaraderie et complicité, et une grande compatibilité sexuelle ${ }^{18}$. Unless s'accepte, accepte son corps, le valorise et s'en réjouit : «Mon âme, c'est mon corps » déclare-t-elle. Elle raconte : «On passe des samedis comme ça. Et des dimanches. Depuis des mois. Les dernières semaines sont sans pudeur. On a déplacé les lits en plein centre du monde. Souvent le monde n'est pas assez grand, on s'élance partout, dans tous les coins, par terre, je retiens les murs $[\ldots] \gg(U$ 25). Unless conclut avec un enthousiasme joyeux : «In sexis hourra! » (U 25). Lorsque Sherpa part en voyage, Unless est très consciente de son absence. À son retour le couple traverse une crise. Le frère d'Unless s'est suicidé, sa sœur Red semble avoir décroché et Unless est malheureuse sans que Sherpa se sente capable de l'aider; après une séparation douloureuse, Unless finit par comprendre, fait le premier pas, dit à Sherpa son amour et admet: «C'est toujours nos plus proches intimes qu'on fait souffrir les premiers » (U 119). C'est la réconciliation : «Trêve de solitude et puisqu'il faut mourir un peu, Sherpa m'attendait. Corps et esprit, alléluia. La grande réconciliation, la belle dépossession. Les bras ouverts, le souper sur le feu, une bouteille et deux verres remplis aux deux tiers. Et le reste. J'ai volé et Sherpa brillait [...] L'état d'ivresse » (U 141). Puis c'est un autre départ de Sherpa. Une fois de plus Unless se sent abandonnée et malheureuse. Mais Sherpa revient vite; il s'est trop ennuyé. Le roman se termine sur une fête, celle de la « réunification des morceaux» (U 185) et Unless conclut : « Si une fille à moitié sourde et démontée connaît les bras où aller, il n'y a aucune raison pour qu'elle se sente dingue » (U 188).

\section{3. Éléna et Pietro (Le bout de la terre)}

Après tout un défilé de couples terriblement mal assortis, malheureux, vers la fin du roman nous trouvons une belle histoire d'amour, celle de Pietro et d'Éléna. Mais nous apprenons en même temps que Pietro est mort noyé et que cette belle histoire d'amour n'a plus d'avenir. Quelques jours après sa mort, Éléna part, quitte l'endroit où ils se sont connus et aimés, car rester là est trop triste pour elle. Ce qui était spécial pour ce couple c'est « qu'ils voulaient changer des choses, améliorer des choses [...] 》 (BT 268) dans

cet endroit si pauvre. Dans une lettre poétique écrite avant sa mort à son ami Alex, Pietro explique comment il est tombé amoureux d'Éléna. Il parle avec adoration de sa luminosité et surtout de sa grande générosité : «Elle est lumineuse, tout simplement. Cette femme a tellement à donner que je crois qu'aucune demande, si grande soit-elle, ne pourrait en venir à bout [...] C'est qu'elle semble avoir trouvé l'accès à une source inépuisable à l'intérieur d'elle-même, une source que ne tarissent ni la fatigue ni l'excès de la demande, une source infinie » (BT 276-277). Grâce à cet amour qu'il ressent, Pietro reconnaît une

http://www.brocku.ca/cfra/voixplurielles06-01/index.html 
double appartenance et aussi une double responsabilité. Il a trouvé un endroit où s'arrêter et quelqu'un à aimer. Pietro, grâce à son amour pour Éléna, a pu dépasser son exil intérieur et sa déchirure. Il explique : «Près d'elle j'ai la très étrange sensation de rentrer chez moi » (BT 277). Il est intéressant que cet amour parfait, cet amour idéal soit rompu brutalement par la mort; est-ce parce que Pietro a terminé son errance et sa quête, et a appris ce qu'il y avait à apprendre? Est-ce parce que ce couple idéal n'aurait pas pu survivre dans le monde « réel » (même fictif) du roman?

\section{Joe et Lise Plamondon (Un sourire blindé)}

Un sourire blindé est un roman mais c'est aussi un conte de fées dans ce qu'il a d'horrible et d'invraisemblable à la fois. Le roman commence par « Il était une fois un petit garçon appelé Conrado » (SB 13) et se termine par « $C^{\prime}$ est parce que je suis si laid, pensa le petit canard en fermant ses yeux» (SB 257). C'est dans le cadre d'un conte de fées que I'histoire tragique du petit Conrado se termine sur une note pleine d'espoir. Conrado, abandonné de son père puis de sa mère, placé dans des familles d'accueil successives se retrouve en prison et, miraculeusement, un couple, Joe et Lise Plamondon, réussissent à le sortir de la prison et à l'adopter. Joe et Lise sont un couple improbable; il est camionneur, elle est serveuse, tous les deux d'Abitibi, d'un village de mineurs. Le petit Conrado, en les voyant pense à ... Yosemite Sam et à Miss Piggy (SB 232-233). Ils ne sont pas riches mais ils mènent une vie heureuse à la campagne. Ils travaillent et pour s'amuser vont danser ou encore à la pêche (SB 238). Mais ce qui est remarquable est leur attachement tendre et généreux à Conrado. Ils s'aiment I'un I'autre et ils aiment Conrado. Ils sont fiers I'un de l'autre aussi et sont fiers de l'enfant. Lise et Joe sont d'une grande discrétion, très respectueux de l'enfant, émouvants et sensibles. Tous les deux sont ouverts et sincères, directs et honnêtes à la fois. Pour pouvoir adopter Conrado ils sont questionnés par les travailleuses sociales; nous apprenons que leurs réponses aux questions concernant leurs habitudes sexuelles et leurs attitudes par rapport au racisme sont « franche[s], candide[s] et d'une [grande] gaillardise» (SB 259). Le narrateur conclut: « c'était des adultes qui savaient se battre et protéger ceux qu'ils aimaient sans rien demander d'autre que la sincérité $[\ldots] \gg($ SB 252-253).

Notons toutefois que les couples modèles proposés sont problématiques à un niveau ou à un autre; le couple « modèle » de La danse juive est critiqué comme conventionnel et conformiste par la narratrice; le couple Unless et Sherpa semblent traverser une crise après l'autre; le couple idéal Éléna et Pietro n'a pas d'avenir, car Pietro meurt très vite; seul le couple idéal Lise et Joe, dans Un sourire blindé, vivra à jamais car il s'agit d'un couple tiré d'un conte de fées... où tout est possible, même les histoires d'amour heureux.

http://www.brocku.ca/cfra/voixplurielles06-01/index.html 
Nous avons examiné le couple dans quelques romans québécois récents; nous avons noté les préoccupations changeantes selon l'âge des partenaires, l'impermanence, la précarité du couple, la violence tant dans la société qui l'entoure qu'au sein du couple lui-même.

Malgré les tensions et les difficultés, malgré l'alcoolisme, la fragmentation et la peur, nous avons pu cerner quelques couples « modèles», couples qui malgré leurs faiblesses et leurs limitations tendent vers un engagement durable et vers la stabilité harmonieuse. Leur camaraderie, leur humour et leur joie d'être ensemble constituent leur force. Ces couples semblent présenter quelques caractéristiques communes; ils se respectent, ils s'aiment tendrement et avec (com)passion et ils sont infiniment généreux l'un avec I'autre, et avec ceux qui les entourent. Leur foi inébranlable en leur couple et en leur engagement soustendent leurs rapports. 
${ }^{1}$ Voir à ce propos Gérard Genette, Figures III, Paris, Éditions du Seuil, p. 238-243.

2 Ying Chen, Les lettres chinoises (Édition princeps: Montréal, Leméac, 1993); nous citons l'édition remaniée : Montréal, Leméac, 1999 (sigle : LC).

3 Monette, Hélène, Unless, Montréal, Boréal, 1995 (sigle : U).

4 Turcotte, Élise, Lîle de la merci, Montréal, Leméac, 1997 (sigle : IM).

${ }^{5}$ Bouyoucas, Pan, La vengeance d'un père, Montréal, Éditions Libre Expression, 1997 (sigle : VP).

${ }^{6}$ Farhoud, Abla, Le bonheur a la queue glissante, Montréal, L'Hexagone, 1998 (sigle : BQG).

7 Muckle, Yan, Le bout de la terre, Montréal, Boréal, 1998 (sigle : BT).

${ }^{8}$ Kokis, Sergio, Un sourire blindé, Montréal, XYZ éditeur, 1998 (sigle : SB).

9 Tremblay, Lise, La danse juive, Montréal, Leméac, 1999 (sigle : DJ).

${ }^{10}$ Marchand, Jacques, Les vents dominants, Montréal, L'Hexagone, 1999 (sigle : VD).

${ }^{11}$ Poitras, Marie Hélène, Soudain le Minotaure, Montréal, Les éditions Triptyque, 2002 (sigle : SLM).

12 À propos du divorce des parents dans Les vents dominants et dans La danse juive André Brochu remarque : «Les parents de Guillaume comme ceux de la narratrice [de La danse juive] ont divorcé, ce qui a laissé de profondes blessures camouflées, mais faciles à reconnaître chez l'un et l'autre » dans «Le meurtre, point final », Lettres québécoises, numéro 96, hiver 1999, p. 20.

${ }^{13}$ De trois hommes ou de trois femmes chez les couples homosexuels, que nous ne retrouvons pas dans notre corpus.

14 Voir à ce propos le premier chapitre de l'ouvrage de René Girard, Mensonge romantique et vérité romanesque, Paris, Grasset, 1961. Ce premier chapitre, intitulé «Le désir 'triangulaire' », examine les écarts et les tensions qui séparent le sujet désirant, le médiateur et l'objet du désir.

15 Voir à ce propos I'ouvrage classique de Muriel James et de Dorothy Jongeward Born to Win, Cambridge MA, Da Capo, 1996. Cet ouvrage a été traduit en français sous le titre Naître gagnant, Paris, InterEditionsDunod, 2006. Voir aussi Ian Stewart et Vann Joines, TA Today, Nottingham and Chapel Hill, Lifespace Publishing, 1997.

${ }^{16}$ C'est nous qui soulignons.

17 Voir à ce propos l'article intitulé « Amour familial et conjugalité » dans le Dictionnaire d'éthique et de philosophie morale (dir. Monique Canto-Sperber) Paris, PUF, 1996, p. 51-55.

18 Voir à ce propos l'article intitulé «Amour » et en particulier la référence à Arthur Schopenhauer et à son ouvrage La métaphysique de l'amour dans le Dictionnaire d'éthique et de philosophie morale (dir. Monique Canto-Sperber) Paris, PUF, 1996, p. 33-45.

http://www.brocku.ca/cfra/voixplurielles06-01/index.html 